\title{
Differential Contributions of Caenorhabditis elegans Histone Deacetylases to Huntingtin Polyglutamine Toxicity
}

\author{
Emily A. Bates, ${ }^{1,2}$ Martin Victor, ${ }^{1}$ Adriana K. Jones, ${ }^{2}$ Yang Shi, ${ }^{1}$ and Anne C. Hart ${ }^{1,2}$ \\ ${ }^{1}$ Department of Pathology, Harvard Medical School, Boston, Massachusetts 02115, and 2Massachusetts General Hospital, Center for Cancer Research, \\ Charlestown, Massachusetts 02129
}

\begin{abstract}
Expansion of a polyglutamine tract in the huntingtin protein causes neuronal degeneration and death in Huntington's disease patients, but the molecular mechanisms underlying polyglutamine-mediated cell death remain unclear. Previous studies suggest that expanded polyglutamine tracts alter transcription by sequestering glutamine rich transcriptional regulatory proteins, thereby perturbing their function. We tested this hypothesis in Caenorhabditis elegans neurons expressing a human huntingtin fragment with an expanded polyglutamine tract (Htn-Q150). Loss of function alleles and RNA interference (RNAi) were used to examine contributions of $C$. elegans cAMP response element-binding protein (CREB), CREB binding protein (CBP), and histone deacetylases (HDACs) to polyglutamineinduced neurodegeneration. Deletion of CREB ( $c r h-1)$ or loss of one copy of CBP ( $c b p-1)$ enhanced polyglutamine toxicity in $C$. elegans neurons. Loss of function alleles and RNAi were then used to systematically reduce function of each $C$. elegans HDAC. Generally, knockdown of individual C. elegans HDACs enhanced Htn-Q150 toxicity, but knockdown of C. elegans hda-3 suppressed toxicity. Neuronal expression of $h d a-3$ restored Htn-Q150 toxicity and suggested that $C$. elegans HDAC3 (HDA-3) acts within neurons to promote degeneration in response to Htn-Q150. Genetic epistasis experiments suggested that HDA-3 and CRH-1 (C. elegans CREB homolog) directly oppose each other in regulating transcription of genes involved in polyglutamine toxicity. hda-3 loss of function failed to suppress increased neurodegeneration in hda-1/+;Htn-Q150 animals, indicating that HDA-1 and HDA-3 have different targets with opposing effects on polyglutamine toxicity. Our results suggest that polyglutamine expansions perturb transcription of CREB/CBP targets and that specific targeting of HDACs will be useful in reducing associated neurodegeneration.
\end{abstract}

Key words: Huntington; polyglutamine; HDAC; polyQ; neurodegeneration; CBP

\section{Introduction}

Huntington's disease (HD) is one of several neurodegenerative diseases caused by expansion of a polyglutamine (polyQ) tract (Zoghbi and Orr, 2000; Ross, 2002). In Huntington's disease, the expansion occurs in the huntingtin protein. Understanding the primary changes that occur in neurons expressing expanded huntingtin could lead to therapeutic interventions. Several hypotheses have been proposed to explain how expansions of polyglutamine domains lead to neurodegeneration. Although, multiple pathways likely contribute to neuronal death, many studies suggest that expanded polyglutamine domains interfere with transcriptional regulation. In patient tissue, as well as in cell culture, vertebrate, and invertebrate models, the expanded polyglutamine form of huntingtin protein binds the acetyltransferase cAMP response element-binding protein (CREB) binding protein (CBP); both huntingtin and CBP are sequestered into aggre-

Received Aug. 9, 2005; revised Dec. 21, 2005; accepted Jan. 25, 2006.

This work was supported by National Institutes of Health-National Institute of Neurological Disorders and Stroke Grant 40048 (A.C.H.). We thank the C. elegans gene knockout consortium (Japan and Oklahoma) and the C. elegans Genetic Center for strains; Mark Alkema and Robert Horvitz for the crh-1(e3315) animals; Beth Muir and Denis Sgroi for assistance with quantitative RT-PCR results; and Johnathan Whetstine, Anders Näär, and members of the Hart and van den Heuvel laboratories for reagents and discussion.

Correspondence should be addressed to Anne C. Hart, Massachusetts General Hospital Center for Cancer Research, 149-7202 13th Street, Charlestown, MA 02129. E-mail: hart@helix.mgh.harvard.edu.

DOI:10.1523/JNEUROSCI.3344-05.2006

Copyright $\odot 2006$ Society for Neuroscience $\quad$ 0270-6474/06/262830-09\$15.00/0 gates (McCampbell et al., 2000; Nucifora et al., 2001; Steffan et al., 2001; Jiang et al., 2003). Moreover, acetyltransferase activity is reduced in cell culture models of Huntington's disease (Nucifora et al., 2001). Increased CBP expression restores histone acetylation, altered transcription, and retinal degeneration in a Drosophila model of polyglutamine disease (Taylor et al., 2003), supporting the hypothesis that CBP is a critical target of expanded polyQ tracts.

Histone acetyltransferase activity is directly opposed by histone deacetylases (HDACs), which remove acetyl groups from core histones. HDACs are divided into three classes based on structure and function. Class I and class II HDACs are similar in sequence and are part of numerous repressor complexes (Grozinger et al., 1999; Knoepfler and Eisenman, 1999). Class III HDACs share little homology with class I and class II HDACs and are homologous to the NAD+-dependent yeast HDAC, SIR2 (silent information regulator 2) (Brachmann et al., 1995), and are implicated in chromatin silencing, cellular metabolism, and aging (Guarente, 2000; Tanner et al., 2000). HDAC proteins have diverse functions and targets, suggesting that inhibition of individual proteins could have different effects on neuronal survival and resistance to polyglutamine toxicity.

Chemical inhibitors of class I and class II HDACs reduce degeneration in cell culture, yeast, Caenorhabditis elegans, and Drosophila models of polyglutamine toxicity (McCampbell et al., 
2001; Steffan et al., 2001; Hockly et al., 2003; Parker et al., 2005). One HDAC inhibitor, suberoylanilide hydroxamic acid, partially rescues motor defects in a mouse model of Huntington's disease (Hockly et al., 2003). However, chemical inhibitors of enzymes are not necessarily specific. Most HDAC inhibitors indiscriminately target class I and class II HDACs, complicating the identification of critical targets.

Our C. elegans model expresses exon 1 of human Htn with an expanded polyglutamine (Htn-Q150) reminiscent of the R6/2 mouse model of HD (Mangiarini et al., 1996; Faber et al., 1999). Expression of Htn-Q150 in the ASH sensory neurons causes progressive neurodegeneration. As in patients, aggregation of mutant protein and neurodegeneration are directly related to the length of the polyglutamine tract and age of the animal (Faber et al., 1999). We found that loss of CBP and CREB function increases polyglutamine toxicity. We surveyed C. elegans HDACs to address possible differential contributions to Htn-Q150 toxicity in neurons and found that $C$. elegans HDACs have divergent effects on polyglutamine toxicity.

\section{Materials and Methods}

RNA interference. To target individual C. elegans HDACs and decrease off-target effects, unique fragments of HDAC exonic sequences were amplified by PCR from genomic DNA using primers listed in the supplemental material (supplemental Table 1, available at www.jneurosci.org). Restriction sites were designed into primers when necessary for cloning into the L4440 plasmid (Timmons et al., 2001). Constructs were amplified in DH5 $\alpha$ cells, and HT115 bacteria were transformed to yield strains for feeding RNA interference (RNAi). RNAi feeding strains for $h d a-1$ (C53A5.3), $h d a-2$ (C08B11.2), and $h d a-3$ (R06C1.1) were provided by Iva Greenwald (Columbia University, New York, NY) (Jarriault and Greenwald, 2002). RNAi feeding strain for $h d a-10$ (Y51H1A.5) was provided by Mark Vidal (Harvard Medical School, Boston, MA). All bacteria feeding strains were cultured as described previously to induce expression of double-stranded RNA (dsRNA) (Timmons et al., 2001). Gravid adult hermaphrodites were lysed using a $20 \%$ bleach and $2.5 \% 10 \mathrm{~N}$ $\mathrm{NaOH}$ solution to release eggs of similar ages. Larva (stage L1) from these egg preparations were placed onto RNAi bacterial strains and passaged to new RNAi plates daily starting at young adult stage. On the eighth day, animals were washed off plates with M9 buffer, dye filled with dioctadecyl tetramethylindodicarbocyanine (DiD) (Perkins et al., 1986), and allowed to recover for at least $20 \mathrm{~min}$ before scoring.

To confirm ASH neuron sensitivity to RNAi, green fluorescent protein (GFP) fluorescence was visually assessed in rtIs11[osm-10::Htn-Q150, osm-10::GFP] young adult animals that were fed bacteria expressing GFP dsRNA from L1 stage. In addition, degeneration of ASH neurons was assessed in 8-d-old aged rtIs11[osm-10::Htn-Q150, osm-10::GFP] fed bacteria expressing pqe-1 (Faber et al., 2002) dsRNA starting at L1 stage.

Strains and aging. Animals were cultured at $25^{\circ} \mathrm{C}$ using standard methods unless otherwise indicated (Brenner, 1974). rtIs18[Htn-Q150], rtIs14[Htn-Q150], and rtIs11[Htn-Q150] transgenic animals express osm-10::Htn-Q150 as described previously (Faber et al., 1999). sir2.1(OEx) are animals that carry the integrated array geIn3 [sir-2.1] (Tissenbaum and Guarente, 2001). deg-1(rt70) (Faber et al., 2002) animals were aged to $4 \mathrm{~d}$ at $20^{\circ} \mathrm{C}$ when $9 \pm 4 \%$ of the ASH neurons degenerate. For each genetic strain generated, control lines [lacking loss of function (lf) mutation or Htn-Q150 transgene] were scored in parallel. To look for suppression of necrotic neuronal death, deg-1 $(r t 70)$ animals were aged to $7 \mathrm{~d}$ at $15^{\circ} \mathrm{C}$ when $78 \pm 4 \%$ of the ASH neurons degenerate. After reaching reproductive maturity (day 3 ), all animals were transferred to new plates daily until the day of scoring. The day of scoring was selected to maximize detection of suppression or enhancement. Each experiment consists of at least three separate days of scoring that total 150 or more neurons when combined.

Overexpression and rescue lines. srb-6::hda-1 (pHA\#442) was constructed by inserting $h d a-1$ cDNA between the KpnI and SacI sites behind the srb-6 promoter of plasmid pHA\#355 (Baran et al., 1999). hda-
1(e1795)/unc-76(e911);rtIs18[Htn-Q150] animals were injected with 50 $\mathrm{ng} / \mu \mathrm{l}$ srb-6::hda-1and $25 \mathrm{ng} / \mu \mathrm{l}$ myo-2::GFP (pPD48.33) (Thatcher et al., $1999)$ to rescue $h d a-1$ (e1795) polyglutamine toxicity enhancement. $h d a-$ 1(e1795);Htn-Q150 homozygous animals carrying the extrasomal srb-6::hda-1 transgene were selected and aged to $6 \mathrm{~d}$ before scoring for degeneration. As a control, hda-1(e1795)/unc-76(e911);rtIs18[HtnQ150] animals were injected with $50 \mathrm{ng} / \mu \mathrm{l} \mathrm{srb-6::empty} \mathrm{pHA} \# 352$ and 25 $\mathrm{ng} / \mu \mathrm{l}$ myo-2::GFP (pPD48.33) and scored in parallel. rtIs18[Htn-Q150]

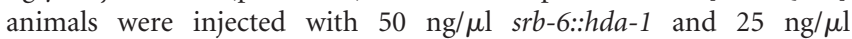
myo-2::GFP to create $h d a-1(\mathrm{OEx})$;Htn-Q150 transgenic lines overexpressing $h d a-1$. srb-6::hda-3 (pHA\#401) rescue constructs were generated by insertion of $h d a-3$ cDNA into pHA\#355 plasmid between the $K p n I$ and SacI sites and injected at $30 \mathrm{ng} / \mu \mathrm{l} s r b-6:: h d a-3$ pHA\#401 with $120 \mathrm{ng} / \mu \mathrm{l}$ pha-1 pBX\#1 into hda-3;pha-1;rtIs11[Htn-Q150] animals. pha-1 rescue serves as a transformation marker. hda-3 overexpression lines were generated by injection of $100 \mathrm{ng} / \mu \mathrm{l} s \mathrm{sb}-6:: h d a-3$ and $120 \mathrm{ng} / \mu \mathrm{l}$ pha-1 pBX\#1 rescue construct in hda-3(+);pha-1;Htn-Q150 animals. At least three transgenic lines were scored and pooled for all genotypes.

Chemical inhibition of HDAC function. pha-1;rtIs11 [Htn-Q150] L1 animals were raised in a humidified chamber at $25^{\circ} \mathrm{C}$ in S-basal medium with (2\%) DMSO with or without $150 \mu \mathrm{g} / \mathrm{ml}$ trichostatin A (TSA) (Sigma, St. Louis, MO) for $8 \mathrm{~d}$ before scoring.

Quantitative PCR. Gravid adults were lysed to collect staged eggs. Embryos were hatched and synchronized to stage L1 larva by starvation overnight in M9 buffer (Johnson et al., 1984). Animals were plated on RNAi nematode growth media plates seeded with HT115 bacteria expressing double-stranded RNA of the desired target gene. Animals developed to young adults at $25^{\circ} \mathrm{C}$. A subset of the adults was aged to confirm degeneration phenotypes. The remaining young adult animals were washed off plates with M9 buffer. After centrifugation, supernatant was replaced with $4 \times$ volume RNAzol (Iso-Tex Diagnostics, Friendswood, $\mathrm{TX})$. Solution was vortexed and flash frozen in liquid N2, allowed to thaw at $37^{\circ} \mathrm{C}$, vortexed, and flash frozen again. This process was repeated twice before tubes were stored at $-80^{\circ} \mathrm{C}$. RNA was isolated using standard procedure by freeze/thaw in RNAzol and phenol/chloroform/isoamylalcohol extraction. cDNA was generated using polyA primers and Super II single-strand reverse transcription (RT)-PCR (Invitrogen, San Diego, CA). RNA was quantified using TaqMan quantitative PCR (PE Biosystems, Foster City, CA). Primers were designed to span sequence from two exons to prevent genomic DNA amplification. Htn-Q150 transcripts were amplified using primers: forward, CAAGGTTACAGCTCGACGAGATC; reverse, CCTCCAAGGGTCCTCCTTTG. All primers anneal at 58 or $59^{\circ} \mathrm{C}$. Transcript levels were detected using TaqMan Htn Probe: CCGGGATTGGCC. Transcripts of two neuronally expressed genes, osm- 6 and cat-1, were measured as comparisons for loading controls (Perkins et al., 1986; Zhang et al., 2004). osm-6 primers are as follows: forward primer, GCCGGAAATTAATGATTACACAAA, and reverse primer, CAATTCTCCTTCGTACATACAAACCTT, which anneals with a melting temperature of $59^{\circ} \mathrm{C}$. osm- 6 transcript levels were detected with TaqMan Probe: TCATATGTCCCAGCAGAT. cat-1: forward primer, TCCGGACCACTTGTCAAG AGT; reverse primer, CTGATGACGGCAATGATGTACA. cat-1 transcript levels were measured with Taqman probe: TAGGATTCCCAACGATG. The reaction contained $5 \mathrm{~nm}$ template DNA and $50 \mu \mathrm{m}$ primer DNA in $50 \mathrm{~mm}$ salt and $1 \mathrm{~mm} \mathrm{Mg}^{2+}$. A three-step PCR was performed for 35 cycles. Denaturation temperature was $94^{\circ} \mathrm{C}$ for $20 \mathrm{~s}$. Annealing was performed at $55^{\circ} \mathrm{C}$ for $20 \mathrm{~s}$. Extension was performed at $72^{\circ} \mathrm{C}$ for $30 \mathrm{~s}$.

Statistical analysis. Error bars represent the SD for multiple determinations of each genotype; each genotype was examined in independent experiments undertaken on more than $1 \mathrm{~d}$ as indicated in the text. $n>$ 150 neurons for each genotype. Pairwise analysis using the two-tailed $t$ test or ANOVA were used as indicated to determine statistical significance. Microsoft Excel (Microsoft, Seattle, WA) was used for all statistical calculations.

\section{Results}

Htn-Q150 expression perturbs CBP-1/CRH-1 function

Previous studies have suggested that expanded polyglutamine tracts perturb CBP and/or CREB function, thereby contributing 


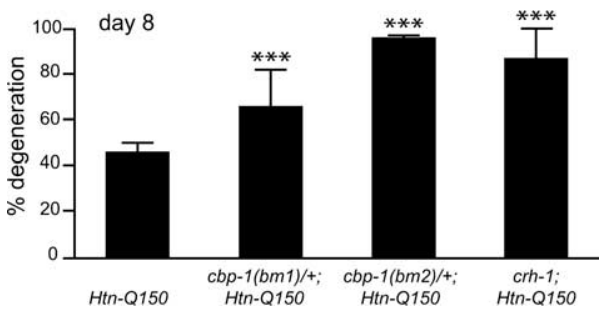

Figure 1. $\quad c b p-1(y s 2) /+, c b p-1(y s 4) /+$, and $c r h-1(3315)$ enhance Htn-0150-induced degeneration. Sibling controls for cbp-1(ys2)/+;Htn-0150, cbp-1(ys4)/+;Htn-0150, and crh1(e3315); Htn-Q150 were combined, because degeneration levels were not significantly different. No degeneration was detected in $c b p-1(y s 2) /+, c b p-1(y s 4) /+$, or $c r h-1$ animals that did not express Htn-0150. Error bars represent SD. ${ }^{* *} p<0.005$ versus column 1 . At least three trials were conducted for each experiment to total $n>150$.

to transcriptional dysregulation and eventual neuronal death. Based on these findings we hypothesized that decreasing CBP or CREB function in neurons expressing an expanded huntingtin protein should increase neurodegeneration. This hypothesis was tested in a C. elegans model of neuronal polyglutamine toxicity (Faber et al., 1999). The first exon of human huntingtin was expressed in a small subset of $C$. elegans neurons, including the two ASH sensory neurons. Each ASH neuron has a sensory process that directly contacts the environment allowing neurons to take up the lipophilic, fluorescent dye DiD. Perturbations in the sensory process prevent dye uptake.

Expression of expanded polyQ tracts leads to neuronal dysfunction, perturbations in ASH sensory processes, and, eventually, cell death. Therefore, dye filling is a rapid, sensitive assay that can detect either degeneration or neuronal death caused by polyQ toxicity; this study does not distinguish between them.

To test the hypothesis that transcriptional dysregulation contributes to polyglutamine toxicity, the requirement for $c b p-1$ function was examined. Each of the two deletion alleles of the $C$. elegans ortholog of CBP, $c b p-1$, were introduced to $\mathrm{rtIs} 18$ [HtnQ150] transgenic animals. $c b p-1(b m 1)$ generates a stable, truncated protein that lacks the conserved histone acetyltransferase (HAT) domain and bromodomain. The $c b p-1(b m 2)$ allele deletes the promoter and first four exons of $c b p-1 . c b p-1(b m 2)$ is a complete loss of function deletion allele; no CBP-1 protein is detected by immunostaining in $c b p-1(b m 2)$ homozygous embryos. Both deletion alleles cause embryonic lethality when homozygous, whereas animals that are heterozygous for $c b p-1$ deletion alleles are indistinguishable from wild-type animals in morphology and viability (Victor et al., 2002). Therefore, Htn-Q150 animals carrying one wild-type $c b p-1$ allele and one $c b p-1$ deletion allele are compared with $H t n-Q 150$ animals with normal $c b p-1$ function (two wild-type alleles). By $8 \mathrm{~d}$, expression of $\mathrm{Htn}$-Q150 causes $46 \pm 4 \%$ of the ASH neurons to degenerate (Fig. 1) (all error bars presented are SDs). Neurodegeneration increased in Htn-Q150; $c b p-1(b m 1) /+$ animals $(67 \pm 16 \%)$ and in Htn-Q150; cbp$1(\mathrm{bm} 2) /+$ animals $(97 \pm 1 \%)$. Both CBP mutant alleles increased Htn-Q150 toxicity, suggesting that CBP-1 HAT activity is critical for protection against polyglutamine toxicity. This is consistent with the hypothesis that expanded polyglutamine tracts interfere with CBP-1-mediated transcriptional regulation.

CREB is one of the DNA binding proteins that interacts with CBP to activate transcription. Reducing CBP-1 function increased polyglutamine toxicity in $C$. elegans, suggesting that CREB function may also be important. To test this hypothesis, we examined the effect of CREB loss of function on Htn-Q150 toxicity. A loss of function deletion allele of a C. elegans CREB ho- molog, crh-1(n3315), resulted in homozygous viable animals with no overt developmental defects (Alkema and Horvitz, manuscript in preparation). Loss of crh-1 function enhanced Htn-Q150-mediated degeneration to $86 \pm 13 \%$ in $\mathrm{Htn}$-Q150 animals versus $45 \pm 8 \%$ in control $\mathrm{Htn}$-Q150 lines (Fig. 1). These results are consistent with the hypothesis that polyglutamine expansions perturb CBP and CREB function.

If polyglutamine expansions cause neuronal degeneration solely by decreasing CBP and CREB function, then reducing function of either CBP or CREB individually could cause degeneration independent of Htn-Q150 expression. To test this, ASH degeneration was assayed in $c b p-1$ and $c r h-1$ homozygous mutant animals lacking the Htn-Q150 transgene. No degeneration was observed in heterozygous $c b p-1(b m 1)$, heterozygous $c b p-1(b m 2)$ animals nor in homozygous crh-1 (n3315) animals in the absence of Htn-Q150 transgene $(n=120)$. Decreasing CBP or eliminating CREB function in C. elegans increases polyglutamine-induced degeneration, but loss of their function is not sufficient to cause degeneration independent of Htn-Q150 expression. This suggests that other cellular pathways are perturbed by polyglutamine expansion. Combined, these data suggest that transcriptional dysregulation is a critical factor in Htn-Q150 toxicity, consistent with previous results suggesting that expanded polyglutamine tracts interfere with CBP-mediated transcription.

\section{Chemical inhibition of class I and class II HDACs decreases degeneration}

Pharmacological manipulation of HDACs alters polyQ toxicity. Sirtuins increase class III HDAC activity and suppress polyQ toxicity in many organisms. Broadly acting class I and class II chemical HDAC inhibitors decrease polyQ-mediated neurodegeneration in vertebrate and Drosophila neurons (McCampbell et al., 2001; Steffan et al., 2001; Hockly et al., 2003). We found that TSA, a chemical inhibitor of class I and class II HDACs, reduces degeneration in C. elegans neurons. pha-1;rtIs11 [Htn-Q150] L1 animals were cultured for $8 \mathrm{~d}$ with or without TSA. Degeneration was reduced from $61 \pm 9 \%$ in control animals to $26 \pm 3 \%$ in animals incubated with $150 \mu \mathrm{g} / \mathrm{ml}$ TSA ( $n>150$ neurons; two trials) (supplemental Fig. 1, available at www.jneurosci.org as supplemental material). TSA suppression was dose dependent; degeneration was moderately reduced by $100 \mu \mathrm{g} / \mathrm{ml} \mathrm{TSA}$ (41 \pm $6 \% ; n=100)$. These results confirm HDACs modulate polyQ toxicity in C. elegans, but broadly acting HDAC inhibitors cannot reveal the contributions of specific HDACs to polyQ toxicity.

\section{Decreasing HDAC gene function has differing effects on Htn-Q150 toxicity}

The activity of histone acetyltransferases, including CBP, is opposed by histone deacetylases. Each of the three HDAC classes is represented in C. elegans (supplemental Fig. 2 and supplemental Table 2, available at www.jneurosci.org as supplemental material). Class I HDACs include hda-1 (C53A5.3), hda-2 (C08B11.2), and hda-3 (R06C1.1) (Shi and Mello, 1998). Mammalian orthologs of $C$. elegans class I HDACs are ubiquitously expressed and predominantly localized to the nucleus. Previous analysis revealed a role for $h d a-1$ in development. Homozygous hda-1(e1794) animals are defective in gonadogenesis and are, consequently, sterile (Dufourcq et al., 2002). C. elegans class II HDACs include $h d a-4$ (C10E2.3), hda-5 (F43G6.11), hda-6 (F41H10.6), hda-10 (Y51H1A.5), and hda-11 (C35A5.9) (Dufourcq et al., 2002). C. elegans class III HDACs include sir-2.1 (R11A8.4), sir-2.2 (F46G10.7), and sir-2.3 (F46G10.3). Decreased function of sir-2.1 reduces lifespan, whereas overexpression of 


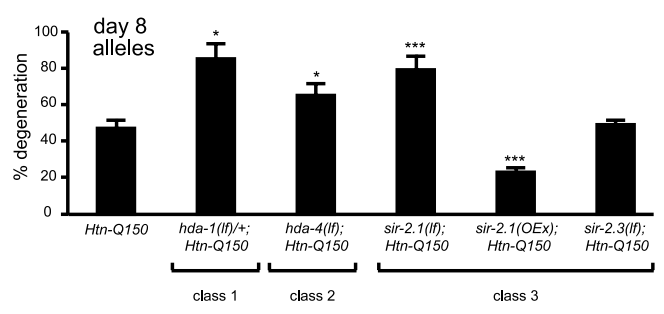

B.

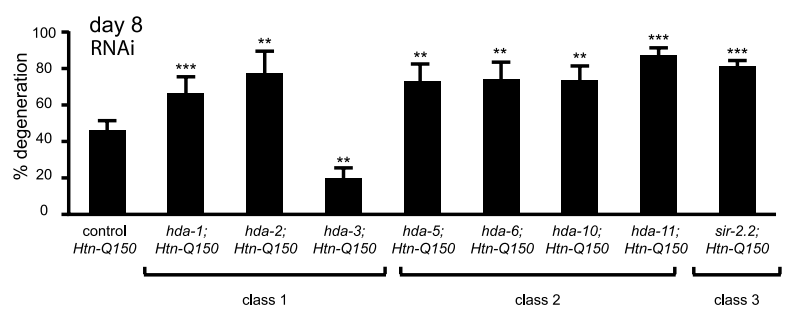

Figure 2. $\quad \boldsymbol{A}$, hda-1(e1795)/+, hda-4, sir-2.1(ok434) enhance Htn-0150 toxicity. sir-2.1 overexpression suppresses Htn-0150 toxicity. sir-2.3 has no effect on Htn- 0150 toxicity. Controls were combined, because they are not significantly different. No degeneration was detected in hda-1(e1795)/+, hda-4, sir-2.1(ok434), or sir-2.3 animals without expression of HtnQ150. At least three trials were conducted for each experiment to total $n>150$. B, RNAi of class I HDACs, hda-1 and hda-2, enhances Htn-0150 toxicity. RNAi of class I HDAC, hda-3, suppresses Htn-Q150 toxicity. RNAi of class II HDACs enhanced degeneration, as did RNAi of sir-2.2, a class III HDAC. Controls were combined, because they are not significantly different. At least three trials were conducted for each experiment to total $n>150$. Error bars represent SD. ${ }^{*} p<0.05$, ${ }^{* *} p<0.005,{ }^{* * *} p<0.0005$ in pairwise comparison with column 1 using a two-tailed $t$ test.

sir-2.1 increases lifespan (Tissenbaum and Guarente, 2001). The relative contribution of these HDACs to polyglutamine toxicity is unclear. Perturbation or modulation of individual HDAC genes could have different effects on polyglutamine toxicity.

In $C$. elegans, gene function can be systematically reduced using either lf alleles or RNAi. Only a few loss of function alleles exist for HDAC genes; RNAi knockdown was used to examine the other $C$. elegans HDAC genes. Although neurons are relatively insensitive to RNAi, we found that feeding animals bacteria expressing dsRNA reduced expression of genes in the ASH neurons. When animals were fed bacteria expressing GFP dsRNA, GFP expression was lost in $88 \%$ of the ASH neurons $(n=100)$, whereas all control animals expressed GFP in both ASH neurons. In addition, when function of a previously described polyQ enhancer-1 ( pqe-1) (Faber et al., 2002) was reduced by feeding pqe-1 dsRNA, Htn-Q150-induced ASH neuron degeneration was increased from $26 \pm 13 \%$ in control animals to $81 \pm 9 \%(n=100$ for each). Thus, ASH neurons are susceptible to RNAi. Loss of function alleles and/or RNAi was used to examine the ability of each C. elegans HDAC gene to modulate polyglutamine toxicity.

Four HDAC loss of function alleles were tested for their ability to modify Htn-Q150 toxicity; three of the four enhanced toxicity. Heterozygous loss of $h d a-1$ increased degeneration of ASH neurons in Htn-Q150 animals ( $86 \pm 6$ from $46 \pm 4 \%$ in control animals at day 8 ) (Fig. 2A). Similarly, reducing function of a class II HDAC, $h d a-4$, increased degeneration $(65 \pm 7 \%)$. Deletion alleles exist for two of the class III HDAC genes (Tissenbaum and Guarente, 2001; Parker et al., 2005). Although complete loss of sir-2.3 function had no effect on Htn-Q150-induced degeneration (48 $\pm 2 \%$ ), complete loss of sir-2.1 function enhanced degeneration $(79 \pm 6 \%)$. Consistent with this result, sir-2.1 overexpression in sir-2.1(OEx);Htn-Q150) animals suppressed degeneration $(23 \pm 2 \%)$ (Fig. $2 A)$.

RNAi was used to examine the contributions of remaining HDACs, because mutant alleles were not available. Consistent

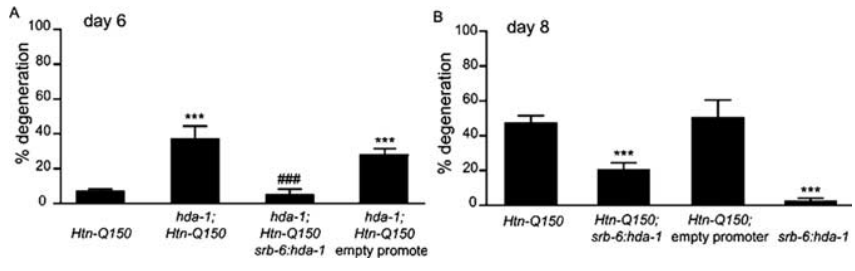

Figure 3. $\quad \boldsymbol{A}$, Expression of HDA-1 in ASH neurons restores Htn-0150 toxicity to normal levels. Injection of equal concentrations of an empty srb- 6 promoter plasmid and the transgenic marker fail to rescue the $h d a-1(e 1795)$ enhancement of polyQ toxicity $(29 \pm 2 \%)$. B, In HtnQ150 animals without the srb- $6:: h d a-1$ transgene, $47 \pm 4 \%$ of the ASH neurons degenerate by day 8 . Degeneration is decreased to $20 \pm 4 \%$ by increased expression of HDA-1 $(50 \mathrm{ng} / \mu \mathrm{l})$ in Htn-0150 ASH neurons. Expression of HDA-1 does not cause detectible degeneration of ASH neurons that do not express $\mathrm{Htn}-\mathrm{O} 150(2 \pm 2 \%)$. No significant change in neurodegeneration is detectable in animals that carry an empty srb- 6 promoter and the transgenic marker myo-2::GFP $(50 \pm 10 \%)$. ${ }^{* * *} p<0.0005$ compared with corresponding Htn-0150 animals in column 1; \#\# $p<0.0005$ when comparing hda-1;Htn-0150; srb-65::hda-1 with hda-1;HtnQ150 in column 2 (to assess rescue) in $\mathbf{A}$. Empty promoter transgenes did not significantly change polyglutamine toxicity. At least three trials were conducted for each experiment to total $n>150$. Error bars represent SD.

with preceding analysis, degeneration is increased to $66 \pm 8 \%$ in hda-1(RNAi);Htn-Q150 animals (from $44 \pm 7 \%$ in Htn-Q150 animals on control RNAi) (Fig. $2 B$ ). Similarly, RNAi of the class I HDAC, $h d a-2$, and all class II HDACs increased degeneration (Fig. 2B) (quantified in supplemental Table 2, available at www. jneurosci.org as supplemental material). In contrast, hda$3(R N A i)$ reduced degeneration $(20 \pm 5 \%)$. RNAi of the class III HDAC, sir-2.2, increased degeneration to $81 \pm 4 \%$. Given the similarity between class I HDACs at the molecular level, the dramatically different effect of $h d a-3$ knockdown is striking. To address the cellular specificity of class I HDACs on polyglutamine toxicity, additional analysis of $h d a-1$ and $h d a-3$ was undertaken.

\section{HDA-1 activity protects Htn-Q150-expressing neurons}

hda-1(e1795) homozygous animals are sterile and die prematurely (Dufourcq et al., 2002). The overall poor health of homozygous $h d a-1(e 1795)$ animals could contribute to the enhanced polyglutamine degeneration observed in hda-1(e1795); Htn-Q150 animals. To address this issue and to determine whether $h d a-1(l f)$ enhancement of polyglutamine toxicity was cell autonomous, an $h d a-1$ cDNA was expressed in ASH neurons using the $s r b-6$ promoter, which drives expression in a limited number of C. elegans neurons (Troemel et al., 1995). Degeneration in homozygous $h d a-1$ (e1795) animals was evaluated at day 6, because $h d a-1$ (e1795) homozygous animals rarely survive longer. In $\mathrm{Htn}-150$ animals, $6 \pm 2 \%$ of the ASH neurons degenerated by day 6 (Fig. 3A). Homozygous loss of $h d a-1$ increases toxicity $(36 \pm 2 \%)$. Expression of the $h d a-1$ cDNA in 6-d-old homozygous $h d a-1(e 1795) ; H t n-Q 150$ animals restored neuronal survival to original levels $(4 \pm 3 \%$ ) (Fig. $3 A$ ). Injection of equal concentrations of an empty srb-6 promoter plasmid, srb-6::empty, and the transgenic marker fail to rescue the homozygous $h d a-$ 1(e1795) enhancement of polyglutamine toxicity ( $29 \pm 2 \%)$. No ASH neurons degenerated in $h d a-1(e 1795)$ homozygous animals lacking the Htn-Q150 transgene. We conclude that loss of hda-1 function is deleterious in neurons that express expanded polyglutamine proteins. Restoring $h d a-1$ levels in a small subset of neurons, including the ASH neurons, is sufficient to ameliorate hda-1 loss of function enhancement of Htn-Q150 toxicity. This suggests that, normally, HDA-1 acts within the affected neuron and can protect against Htn-Q150 toxicity; reducing HDA-1 function sensitizes neurons to polyglutamine assault. 

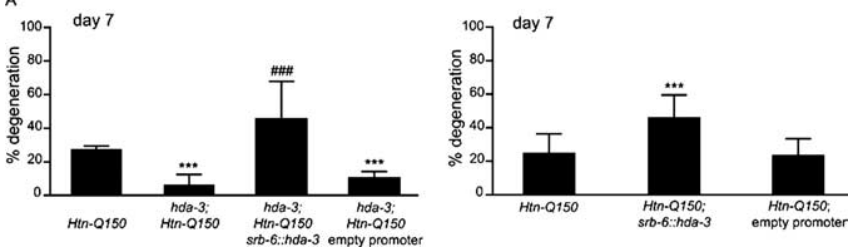

Figure 4. HDA-3 specifically rescues hda-3(tm1374) suppression of Htn-0150 in ASH neurons. Degeneration is reduced from $27 \pm 2 \%$ in rt/s $11 \mathrm{Htn}-0150$ animals to $6 \pm 6 \%$ in hda3(tm1374); rtls11(Htn-0150) animals at day 7. Degeneration ranges from 14 to 63\%, depending on the transgenic line (average, $45 \pm 22 \%$ ) in hda-3(tm 1374); rtls11(Htn-0150); srb-6: hda-3 transgenic animals. As a control, a plasmid carrying the empty srb-6 promoter was injected along with transgenic markers. The empty promoter has no significant effect on polyQ toxicity (10 $\pm 3 \%)$. Overexpression of $h d a-3$ in ASH using the srb- 6 promoter increases degeneration from $24 \pm 11$ to $45 \pm 13 \%(p=0.03) .{ }^{* *} p<0.0005$ compared with column 1 for

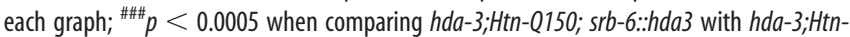
Q150. At least three trials were conducted for each experiment to total $n>150$. Error bars represent SD.

Decreasing $h d a-1$ function could negatively affect neuronal survival by a nonspecific mechanism. However, if overexpression of $h d a-1$ protects against Htn-Q150-induced neurodegeneration, then HDA-1 targets are likely directly involved in protecting polyglutamine-expressing neurons. To test this hypothesis, $h d a-1$ was overexpressed in ASH neurons. Htn-Q150 animals were injected with $s r b-6:: h d a-1$ to create $h d a-1$ overexpression transgenic animals [hda-1(OEx);Htn-Q150]. Suppression is most readily detected by comparing degeneration in Htn-Q150 animals, Htn-Q150; hda-1(OEx) animals, and Htn-Q150; srb-6::empty transgenic control animals at day 8. In Htn-Q150 animals without the $s r b-6:: h d a-1$ transgene, $47 \pm 4 \%$ of the ASH neurons degenerate by day 8 . Degeneration is decreased to $20 \pm$ $4 \%$ by increased expression of HDA-1 in Htn-Q150 ASH neurons (Fig. 3B). Expression of HDA-1 does not cause significant degeneration in the absence of the Htn-Q150 transgene (1 \pm $2 \%)$. Increased HDA-1 activity in the neurons protects them against Htn-Q150 toxicity, suggesting that HDA-1 targets may be critical factors in decreasing polyglutamine toxicity.

\section{HDA-3 promotes degeneration in response to Htn-Q150 expression}

The hda-3(RNAi) analysis suggested that reducing HDA-3 function could decrease Htn-Q150-induced degeneration. To confirm the role of hda-3 in Htn-Q150-induced neurodegeneration, a deletion allele, $t m 1374$, was obtained from the C. elegans Gene Knockout Center (Tokyo, Japan). The tm1374 deletion begins in the second exon of $h d a-3$, omits DNA sequence encoding the FPGTGDL residues that are critical for deacetylase activity (Finnin et al., 1999), and results in a frame shift that is likely to eliminate $h d a-3$ function. The integrated $r$ Is18[Htn-Q150] array maps are very close to the $h d a-3$ gene on chromosome I. Therefore, it was necessary to use a different $\mathrm{Htn}$-Q150 transgene for hda-3(tm1374) analysis. Degeneration was assessed in rtIs11[Htn-Q150] and rtIs14[Htn-Q150] animals on day 7 when either enhancement or suppression of Htn-Q150 toxicity could be detected. hda-3(tm1374) recapitulated the suppression of Htn-Q150 toxicity observed in hda-3(RNAi) animals. Degeneration was reduced from $27 \pm 2 \%$ in rtIs11[Htn-Q150] animals to $6 \pm 5 \%$ in homozygous hda-3(tm1374);rtIs11[Htn-Q150] animals (Fig. 4A).

To confirm that HDA-3 acts in the polyglutamine expressing neurons to modulate toxicity, we examined the HDA-3 cellular expression pattern. Immunohistochemistry revealed that HDA-3 is ubiquitously expressed. Costaining with an OSM-10 antisera that recognizes an endogenous protein expressed in the ASH (Hart et al., 1999) confirmed that HDA-3 protein is found in the ASH neurons. Endogenous HDA-3 predominantly localizes to the nucleus in C. elegans, where it is likely to function in transcriptional regulation (supplemental Fig. 3, available at www. jneurosci.org as supplemental material) (Whetstine et al., 2005).

To determine whether hda-3 modulation of Htn-Q150 toxicity occurs in the neurons and to confirm that reduced $h d a-3$ function is responsible for suppression, an $s r b-6:: h d a-3$ transgene was introduced into hda-3;rtIs11[Htn-Q150] animals. Polyglutamine-induced degeneration of ASH neurons was restored when hda-3 was expressed in a small subset of neurons, including ASH. Degeneration averaged $31 \pm 8 \%$ in hda-3(tm1374);rtIs11[Htn-Q150];srb-6::hda-3(+) transgenic animals compared with $27 \pm 2 \%$ in rtIs11[Htn-Q150] control animals and $10 \pm 3 \%$ in hda-3;rtIs11[Htn-Q150] animals (Fig. $4 A$ ). Thus, decreased HDA-3 activity decreases the toxicity of Htn-Q150 specifically in neurons in which polyglutamine is expressed.

To determine whether HDA-3 contributes directly to HtnQ150-induced degeneration in ASH neurons, srb-6::hda-3 was introduced into Htn-Q150 animals that retain normal hda-3 activity. Overexpression of $h d a-3$ in ASH using the $s r b-6$ promoter increased degeneration from $24 \pm 11$ to $45 \pm 13 \%$ (Fig. $4 B$ ). We concluded that suppression of polyglutamine toxicity in hda3(tm1374);rtIs11[Htn-Q150] animals is attributable to loss of $h d a-3$ function and that $h d a-3$ acts cell autonomously to modulate polyglutamine toxicity in these neurons.

\section{Loss of hda-3 or hda-1 specifically modulates polyglutamine-induced neurotoxicity}

To determine whether $h d a-1$ or $h d a-3$ modulation of neurotoxicity is specific to polyglutamine-induced degeneration, we examined possible roles of $h d a-1$ and $h d a-3$ in necrotic neurodegeneration independent of polyglutamine toxicity. Hyperactive ion channels disrupt cellular osmotic balance and cause necrotic neuronal degeneration. A recessive, cold-sensitive mutation in a sodium channel, deg-1(rt70), causes temperature-dependent ASH degeneration (Faber et al., 2002). $h d a-1$ and $h d a-3$ functions were individually reduced by RNAi in deg-1(rt70) mutant animals. deg-1 (rt70) animals were aged to $4 \mathrm{~d}$ at $20^{\circ} \mathrm{C}$ when $9 \pm 4 \%$ of the ASH neurons degenerate or were aged to $7 \mathrm{~d}$ at $15^{\circ} \mathrm{C}$ when $78 \pm 6 \%$ of the ASH neurons degenerate to assess enhancement or suppression, respectively. Neither hda-1(RNAi) nor hda3(RNAi) altered necrotic degeneration (supplemental Fig. 4, available at www.jneurosci.org as supplemental material). These data suggest that transcriptional dysregulation selectively contributes to polyglutamine-induced degeneration.

\section{Reducing function of $h d a-3$ or $h d a-1$ does not significantly alter Htn-Q150 expression}

Reducing function of specific proteins found in HDAC complexes can alter transgene expression levels in many C. elegans tissues, but neurons are generally protected from these alterations (Kim et al., 2005). To address the possibility that HtnQ150 transgene levels were changed by loss of HDAC function in the ASH neurons, we took three experimental approaches. First, to demonstrate that osm-10::Htn-Q150 transgene levels are not altered by $h d a-3$ loss of function, we quantified expression of osm-10::GFP in homozygous hda-3(tm1374);osm-10::GFP animals. GFP expression in ASH neurons was indistinguishable 


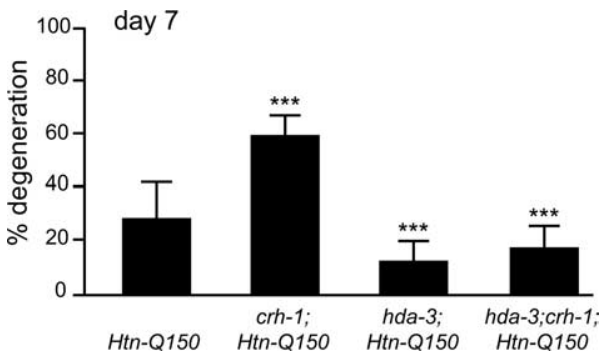

Figure 5. HDA-3 modulates Htn- 0150 toxicity via transcriptional regulation. Loss of $h d a-3$ function suppresses the deleterious effects of loss of $\mathrm{crh}-1$ function. ASH neuron degeneration is increased in crh-1;rtls11[Htn-0150] animals and decreased in hda-3(tm1374);rtls11[Htn-0150] animals compared with rtls 11 [Htn-0150]. ASH degeneration is suppressed in hda-3(tm 1374); crh-1; rtls11[Htn-0150] animals and is not significantly different from degeneration levels in hda-3(tm1374);rtls11[Htn-0150] animals at day 7. Error bars represent SD. ${ }^{* * *} p<0.0005$ compared with column 1 of each graph. At least three trials were conducted for each determination to total $n>150$.

from control osm-10::GFP animals $(n=380)$. To directly determine whether genes implicated in transgene silencing decrease osm-10 promoter activity, we examined GFP expression in 3-dold rtIs11 [osm-10::GFP, osm-10::Htn-Q150] animals lacking an RNA-directed RNA polymerase, $r r f-3$, or lacking a ribosomal RNA repressor, $n c l-1$ (Frank and Roth, 1998; Simmer et al., 2002). Both of these genes are expressed in ASH neurons and have been implicated previously in transgene silencing in C. elegans (Simmer et al., 2002; Kim et al., 2005). Neither rrf3 (pk1426) nor $n c l-1(e 1865)$ silence expression of GFP in ASH neurons ( $n=200$ and $n=150$, respectively). Additionally, to determine specifically whether $r r f-3(p k 1426)$ or $n c l-1(e 1865)$ could suppress or enhance polyglutamine-induced degeneration, ASH neuron degeneration was assessed in corresponding animals. Degeneration was not altered in $r r f-3$ or $n c l-1$ homozygous mutant backgrounds (supplemental Fig. 5A, available at www. jneurosci.org as supplemental material). Therefore, we consider it unlikely that transgene silencing contributes to decreased neurodegeneration in hda-3 mutant animals.

To directly address the possibility that reducing HDAC function alters Htn-Q150 expression levels, consequently altering polyglutamine toxicity, Htn-Q150 mRNA levels were measured using quantitative RT-PCR. Transcript levels were measured in hda-3(RNAi), hda-1(RNAi), and control RNAi rtIs18[Htn-Q150] animals. Levels were assessed in young adult animals before the onset of neuronal degeneration. There was no significant difference in Htn-Q150 mRNA levels (supplemental Fig. 5B, available at www.jneurosci.org as supplemental material). We conclude that changes in Htn-Q150 toxicity are not attributable to changes in Htn-Q150 transcript levels.

\section{HDA-3 loss suppresses CREB modulation Htn-Q150-induced degeneration}

Histone deacetylases are critical for transcriptional regulation, but HDACs are not limited to histones as targets (Hubbert et al., 2002). C. elegans HDACs could modulate polyglutamine toxicity via transcriptional regulation or by deacetylating proteins that are directly involved with polyglutamine toxicity. Genetic epistasis analysis was undertaken to determine whether $h d a-3(t m 1374)$ suppression of Htn-Q150 toxicity is via regulation of CBP/CREB transcription. Double homozygous hda-3;crh-1;Htn-Q150 animals were generated and compared with homozygous hda-3; Htn-Q150, homozygous crh-1;Htn-Q150, and Htn-Q150 sibling lines. If HDA-3 and CRH-1 participate in independent pathways,

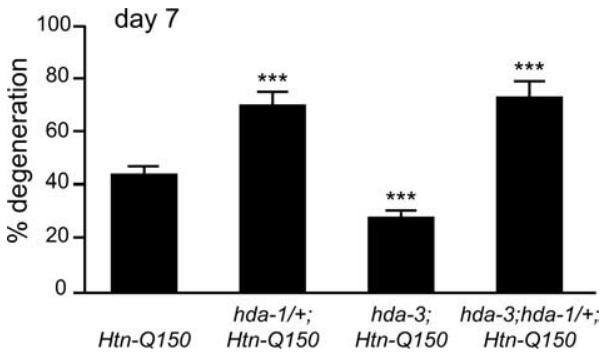

Figure 6. Normal hda-1 levels are critical for hda-3 modulation of polyglutamine toxicity. Loss of one copy of $h d a-1$ eliminates the salubrious effects of $h d a-3$ loss of function. ASH neuron degeneration is increased in hda-1(e1795)/+;rt/s 14[Htn-Q150] animals and decreased in hda3(tm1374);rtls 14[Htn-0150i] animals compared with rt/s14[Htn-0150] animals. Degeneration levels are increased in hda-3(tm1374);hda-1(e1795)/+;rtls14[Htn-0150] and are not significantly different from degeneration levels in hda-1(e1795)/+; rtls14[Htn-0150] animals. Error bars represent SD. ${ }^{* * *} p<0.0005$ compared with column 1. At least three trials were conducted for each determination to total $n>150$.

their combined effects could be additive, resulting in polyglutamine degeneration levels between $c r h-1$ enhanced degeneration and $h d a-3$ suppressed degeneration. If HDA-3 and CRH-1 (C. elegans CREB homolog) act in the same pathway, either the phenotype of $h d a-3$ (suppression) or the phenotype of $c r h-1$ (enhancement) should predominate. $28 \pm 13 \%$ of the ASH neurons degenerate in control Htn-Q150 animals. As expected, degeneration increased to $59 \pm 7 \%$ in $\mathrm{crh}-1 ; \mathrm{Htn}-\mathrm{Q} 150$ animals and decreased by loss of hda-3 to $12 \pm 7 \%$ in Htn-Q150 animas (Fig. 5). Interestingly, hda-3 loss of function suppressed the $\mathrm{crh}-1$ loss of function phenotype in hda-3(tm1374); crh-1;Htn-Q150 animals to $17 \pm 8 \%$. Thus, $h d a-3$ is epistatic to $c r h-1$, suggesting that HDA-3, directly or indirectly, opposes crh-1 activity in regulating transcription of genes that protect against Htn-Q150-induced toxicity.

\section{Suppression of Htn-Q150 toxicity in hda-3 mutant animals requires HDA-1 function}

Reducing hda-1 function enhances Htn-Q150 toxicity. In contrast, reducing $h d a-3$ function suppresses Htn-Q150 toxicity. To determine whether $h d a-3$ loss of function could suppress $h d a-1$ enhanced polyglutamine toxicity, ASH neuron degeneration was assessed in hda-3;hda-1/+;Htn-Q150 animals. As expected, degeneration was reduced from $45 \pm 4 \%$ in control rtIs14[HtnQ150] animals to $29 \pm 2 \%$ in hda-3;rtIs14[Htn-Q150] animals at day 7. Consistent with results described above, degeneration was increased in hda-1/+;rtIs14[Htn-Q150] animals to $76 \pm 6 \%$ (Fig. 6). Interestingly, degeneration was also increased in hda3(tm1374); hda-1(e1795)/+;rtIs14[Htn-Q150] animals to $74 \pm$ $7 \%$ versus controls. $h d a-3$ loss of function can suppress degeneration when $h d a-1$ function is intact but cannot suppress HtnQ150-induced degeneration when hda-1 levels are compromised. One possible interpretation of these results is that HDA-1 activity is required, directly or indirectly, for suppression of HtnQ150 toxicity by hda-3 loss of function.

\section{Discussion}

This analysis suggests that expression of expanded Htn fragments interferes with CBP and/or CREB function, thereby contributing to neuronal degeneration. Reducing function of most $C$. elegans histone deacetylases enhances Htn-Q150-induced degeneration. Additional analysis confirms that HDA-1 protects Htn-Q150 expressing neurons from degeneration. HDA-3 is unique; loss of HDA-3 suppresses polyglutamine toxicity. 
Reducing $c b p-1$ and $c r h-1$ activity enhances Htn-Q150induced degeneration. This suggests that expanded polyglutamine perturbs transcription leading to neuronal degeneration. Histone acetyltransferase and Q-rich domains of human CBP interact with expanded Htn (Steffan et al., 2001). C. elegans cbp$1(b m 1)$, the allele that deletes the histone acetyltransferase and bromodomains, significantly enhances polyglutamine toxicity. $c b p-1(b m 1)$ encodes a stable, truncated CBP-1 protein that lacks acetyltransferase activity (Victor et al., 2002). This suggests that acetyltransferase activity is important for CBP-1 protection against Htn-Q150 toxicity.

Reducing $c r h-1$ (CREB) function is sufficient to enhance polyglutamine toxicity, implicating $c r h-1$ regulated genes in neuronal survival. Deletion of CREB combined with deletion of its cofactor, CREM (cAMP-responsive element modulator), causes a phenotype similar to Huntington's disease in mice (Mantamadiotis et al., 2002). However, reducing C. elegans cbp-1 or eliminating $c r h-1$ function does not cause degeneration independent of the Htn-Q150 transgene. We conclude that Htn-Q150 perturbs additional transcriptional pathways and/or cellular processes that contribute to degeneration. In vertebrates, TATA binding protein, specificity protein 1 , and p53 have all been found in polyglutamine aggregates (Cha, 2000; Sugars and Rubinsztein, 2003). In addition, proteasomes, ubiquitin, and chaperones have been found in aggregates, suggesting disruption of the protein folding and degradation pathways (Sakahira et al., 2002; Ciechanover and Brundin, 2003). The conclusion that multiple pathways contribute to polyglutamine toxicity is strengthened by the recent finding that protective effects of increased CREB and heatshock protein 70 are additive in Drosophila (Iijima-Ando et al., 2005). We conclude that histone acetylation activity of CBP-1 and transcriptional activation by CRH-1 are likely perturbed by Htn-Q150 expression contributing to the process of neurodegeneration.

Chemical and genetic manipulation of HDAC function yield distinctly different results for polyQ toxicity. This likely reflects their different specificity and efficacy. polyQ toxicity is reduced by broadly acting chemical inhibitors of class I and class II HDACs. In contrast, eliminating specific HDACs can increase or reduce degeneration depending on the gene targeted. At first glance, this discrepancy may be puzzling. However, at the concentrations tested, broadly acting chemical inhibitors like TSA likely cause a partial decrement in all class I and class II HDACs. These HDACs likely regulate many genes with various and divergent impacts on polyQ toxicity. It is reasonable to assume that eliminating specific HDACs using genetic techniques might then yield dramatically different results; loss of a specific HDAC likely yields specific and unique changes in gene expression (Dangond and Gullans, 1998). Additionally, chemical or genetic manipulation may differ in their impact on the compensatory mechanisms that operate when HDAC function is decreased (Ajamian et al., 2004). And, chemical inhibitors may have off-target effects, perturbing proteins other than HDACs. Previous analyses using broadly acting chemical inhibitors may have obscured the relative importance of specific HDACs in polyQ toxicity.

Decreasing the function of most class I and class II HDACs increased polyglutamine toxicity in C. elegans. Mammalian $\mathrm{HDAC} 1$ and $\mathrm{HDAC} 2$ are found together in numerous complexes [Sin3, vcleosome remodeling and deacetylation, retinoblastoma, and corepressor to RE1 silencing transcription factor/neural restrictive silencing factor corepressor complexes (Knoepfler and Eisenman, 1999; Humphrey et al., 2001)]. In mammals, Sin3 is important for the regulation of fatty acid synthesis, which is crit- ical for mitochondrial function and vesicle formation, both of which have been implicated in polyglutamine expansion disease (Schweizer and Hofmann, 2004). Sin3 and nuclear repressor protein $(\mathrm{NCoR})$ interact with Htn when the polyglutamine tract is expanded and have been found in polyglutamine protein aggregates (Boutell et al., 1999). Although mammalian HDAC3 is found in complexes with NCoR and SMRT (silencing mediator of retinoic acid and thyroid hormone receptor), it is not yet clear in which complexes C. elegans hda-3 takes part (Guenther et al., 2000, 2001; Li et al., 2000; Wen et al., 2000). If ectopic polyglutamine interactions with $\operatorname{Sin} 3$ and NCoR titrate away functions of these repressor complexes, then reducing the function of HDACs in the Sin 3 and NCoR complexes could further decrease repressor complex function and thereby impinge on neuronal survival.

In this study, reducing function of most class II HDACs increases polyglutamine toxicity. Many class II HDACs have myocyte enhancer factor-2 (MEF-2) interaction domains (Choi et al., 2002). Mammalian MEF-2 proteins play important roles in neuronal survival (Mao and Wiedmann, 1999; Youn et al., 1999). Thus, decreasing function of class II HDACs could compromise survival of neurons. HDAC4 and HDAC5 repression of MEF-2 is dependent on calcium, suggesting a possible link between calcium signaling and polyglutamine toxicity (Youn et al., 2000). Thus, C. elegans HDA-1, HDA-2, and class II HDACs could be important for maintaining survival of neurons under polyglutamine stress.

In contrast, loss of $\mathrm{Ce}$-hda-3 suppresses polyglutamine toxicity. Mammalian HDAC3 is found in different complexes from HDAC1 and HDAC2 (Guenther et al., 2000, 2001; Wen et al., 2000). Thus, specific HDAC complexes could regulate genes critical for protection against polyglutamine toxicity or neuronal survival, whereas other complexes regulate genes that contribute to polyglutamine toxicity.

Class III HDACs have been implicated previously in polyglutamine toxicity and aging. In C. elegans and Drosophila, increased Sir2 function extends the lifespan (Tissenbaum and Guarente, 2001; Wood et al., 2004). In this study, reducing function of two of the three C. elegans class III HDACs enhanced polyglutamine toxicity. Interestingly, Ce-sir-2.1 deletion increases Htn-Q150induced degeneration, whereas overexpression suppresses degeneration. Our results are consistent with recent findings that increasing activity of $\mathrm{Ce}$-sir-2.1 protects against polyglutamineinduced neuronal dysfunction (Parker et al., 2005). Based on the results presented herein, Ce-sir-2.1 is not unique. Decreasing function of most $\mathrm{Ce}$-HDACs increases polyglutamine toxicity, and increasing both $C e-h d a-1$ and $C e$-sir-2.1 suppresses toxicity. The results presented here suggest that SIR-2.1 HDAC activity is critical for polyglutamine toxicity but not necessarily increased longevity or aging associated with activity of sir-2.1.

Is the activity of $C e-h d a-3$ as a transcriptional regulator critical for its role in polyglutamine toxicity? Some HDACs target proteins that are not involved in transcription. For instance, mammalian HDAC6 and Sir2 deacetylate tubulin (Hubbert et al., 2002; Matsuyama et al., 2002; North et al., 2003; Zhang et al., 2003). Therefore, at least two functional targets exist for HDACs in polyglutamine toxicity. First, specific HDACs could regulate the transcription of genes that encode protective proteins or proteins that assist in degeneration. Second, specific HDACs could deacetylate proteins that play nontranscriptional roles underlying polyglutamine toxicity. Based on the ability of $\mathrm{Ce}-\mathrm{hda}-3 \mathrm{de}-$ letion to suppress increased polyglutamine toxicity of $\mathrm{crh}-1 \mathrm{mu}-$ tants, genetic epistasis suggests that HDA-3 modulates polyglutamine toxicity through transcriptional regulation. $\mathrm{crh}-1$ 
loss of function can no longer enhance polyglutamine toxicity in hda-3 mutant animals, suggesting that other transcriptional activators are able to compensate for $c r h-1$ loss of function when hda-3 function is reduced. HDA-3 might normally repress transcription of $C$. elegans genes that protect against expanded polyglutamine or promote neuronal survival. Therefore, release from negative regulation via $h d a-3$ loss of function protects against polyglutamine toxicity.

Here, we demonstrate that normal Ce-hda-1 function is required for Ce-hda-3(tm1374) suppression of Htn-Q150-induced neurodegeneration. There are at least two molecular mechanisms that could underlie alterations in polyglutamine toxicity. $\mathrm{Ce}$ $h d a-1$ protein could negatively regulate cell death genes, and/or HDA-3 could repress transcription of protective genes. One possible interpretation of the epistasis analysis is that $\mathrm{Ce}$-hda-1 target genes are downstream of $\mathrm{Ce}-\mathrm{HDA}-3$ targets. A second possible explanation is that $C e-h d a-1$ transcription may normally be $\mathrm{di}-$ rectly repressed by $\mathrm{Ce}$-HDA-3 protein in ASH neurons. Therefore, when $C e-h d a-3$ function is reduced, Ce-hda-1 transcription is increased. Our results demonstrate that increased Ce-hda-1 dosage suppresses Htn-Q150-induced neurodegeneration. Therefore, Ce-hda-3 loss of function could suppress polyglutamine toxicity by increasing HDA-1 activity. Thus, in Ce-hda3(tm1374);Ce-hda-1(e1795);rtIs14[Htn-Q150] animals, neurodegeneration may not be suppressed, because Ce-hda-1 activity cannot be sufficiently increased. However, we cannot rule out the possibility that $h d a-3(l f)$ cannot compensate for the severity of hda-1(lf) degeneration.

In C. elegans, genes positively regulated by CREB/CBP and negatively regulated by $h d a-3$ likely play important roles protecting against expanded polyglutamine. We speculate that in mammals, specific HDACs could negatively regulate transcription of $b c l-2, \mathrm{BDNF}$, chaperones, or other proteins necessary for neuronal survival, whereas other HDACs regulate transcription of degeneration promoting genes such as caspases.

In summary, using a C. elegans neuronal model of polyglutamine disease, we found that CBP and CREB functions are critical for protecting against polyglutamine-induced neurodegeneration. Results presented here suggest that HDACs play opposing roles in polyglutamine toxicity in C. elegans neurons. Therefore, it is likely that in mammals, specific HDACs will also have opposing effects on polyglutamine-induced degeneration. These $C$. elegans results suggest that finding downstream target genes of specific HDAC complexes will help to determine the mechanism of polyglutamine-induced degeneration. The development of inhibitors targeted to specific HDACs or HDAC complexes could be both more effective and less toxic therapeutic agents in polyglutamine expansion diseases.

\section{References}

Ajamian F, Salminen A, Reeben M (2004) Selective regulation of class I and class II histone deacetylases expression by inhibitors of histone deacetylases in cultured mouse neural cells. Neurosci Lett 365:64-68.

Baran R, Aronoff R, Garriga G (1999) The C. elegans homeodomain gene unc- 42 regulates chemosensory and glutamate receptor expression. Development 126:2241-2251.

Boutell JM, Thomas P, Neal JW, Weston VJ, Duce J, Harper PS, Jones AL (1999) Aberrant interactions of transcriptional repressor proteins with the Huntington's disease gene product, huntingtin. Hum Mol Genet 8:1647-1655.

Brachmann CB, Sherman JM, Devine SE, Cameron EE, Pillus L, Boeke JD (1995) The SIR2 gene family, conserved from bacteria to humans, functions in silencing, cell cycle progression, and chromosome stability. Genes Dev 9:2888-2902.

Brenner S (1974) The genetics of Caenorhabditis elegans. Genetics 77:71-94.
Cha JH (2000) Transcriptional dysregulation in Huntington's disease. Trends Neurosci 23:387-392.

Choi KY, Ji YJ, Jee C, Kim do H, Ahnn J (2002) Characterization of CeHDA-7, a class II histone deacetylase interacting with MEF-2 in Caenorhabditis elegans. Biochem Biophys Res Commun 293:1295-1300.

Ciechanover A, Brundin P (2003) The ubiquitin proteasome system in neurodegenerative diseases: sometimes the chicken, sometimes the egg. Neuron 40:427-446.

Dangond F, Gullans SR (1998) Differential expression of human histone deacetylase mRNAs in response to immune cell apoptosis induction by trichostatin A and butyrate. Biochem Biophys Res Commun 247:833-837.

Dufourcq P, Victor M, Gay F, Calvo D, Hodgkin J, Shi Y (2002) Functional requirement for histone deacetylase 1 in Caenorhabditis elegans gonadogenesis. Mol Cell Biol 22:3024-3034.

Faber PW, Alter JR, MacDonald ME, Hart AC (1999) Polyglutaminemediated dysfunction and apoptotic death of a Caenorhabditis elegans sensory neuron. Proc Natl Acad Sci USA 96:179-184.

Faber PW, Voisine C, King DC, Bates EA, Hart AC (2002) Glutamine/ proline-rich $\mathrm{PQE}-1$ proteins protect Caenorhabditis elegans neurons from huntingtin polyglutamine neurotoxicity. Proc Natl Acad Sci USA 99:17131-17136.

Finnin MS, Donigian JR, Cohen A, Richon VM, Rifkind RA, Marks PA, Breslow R, Pavletich NP (1999) Structures of a histone deacetylase homologue bound to the TSA and SAHA inhibitors. Nature 401:188-193.

Frank DJ, Roth MB (1998) ncl-1 is required for the regulation of cell size and ribosomal RNA synthesis in Caenorhabditis elegans. J Cell Biol 140:1321-1329.

Grozinger CM, Hassig CA, Schreiber SL (1999) Three proteins define a class of human histone deacetylases related to yeast Hdalp. Proc Natl Acad Sci USA 96:4868-4873.

Guarente L (2000) Sir2 links chromatin silencing, metabolism, and aging. Genes Dev 14:1021-1026.

Guenther MG, Lane WS, Fischle W, Verdin E, Lazar MA, Shiekhattar R (2000) A core SMRT corepressor complex containing HDAC3 and TBL1, a WD40-repeat protein linked to deafness. Genes Dev 14:1048-1057.

Guenther MG, Barak O, Lazar MA (2001) The SMRT and N-CoR corepressors are activating cofactors for histone deacetylase 3. Mol Cell Biol 21:6091-6101.

Hart AC, Kass J, Shapiro JE, Kaplan JM (1999) Distinct signaling pathways mediate touch and osmosensory responses in a polymodal sensory neuron. J Neurosci 19:1952-1958.

Hockly E, Richon VM, Woodman B, Smith DL, Zhou X, Rosa E, Sathasivam K, Ghazi-Noori S, Mahal A, Lowden PA, Steffan JS, Marsh JL, Thompson LM, Lewis CM, Marks PA, Bates GP (2003) Suberoylanilide hydroxamic acid, a histone deacetylase inhibitor, ameliorates motor deficits in a mouse model of Huntington's disease. Proc Natl Acad Sci USA 100:2041-2046.

Hubbert C, Guardiola A, Shao R, Kawaguchi Y, Ito A, Nixon A, Yoshida M, Wang XF, Yao TP (2002) HDAC6 is a microtubule-associated deacetylase. Nature 417:455-458.

Humphrey GW, Wang Y, Russanova VR, Hirai T, Qin J, Nakatani Y, Howard BH (2001) Stable histone deacetylase complexes distinguished by the presence of SANT domain proteins CoREST/kiaa0071 and Mta-L1. J Biol Chem 276:6817-6824.

Iijima-Ando K, Wu P, Drier EA, Iijima K, Yin JC (2005) cAMP-response element-binding protein and heat-shock protein 70 additively suppress polyglutamine-mediated toxicity in Drosophila. Proc Natl Acad Sci USA 102:10261-10266.

Jarriault S, Greenwald I (2002) Suppressors of the egg-laying defective phenotype of sel-12 presenilin mutants implicate the CoREST corepressor complex in LIN-12/Notch signaling in C. elegans. Genes Dev $16: 2713-2728$.

Jiang H, Nucifora Jr FC, Ross CA, DeFranco DB (2003) Cell death triggered by polyglutamine-expanded huntingtin in a neuronal cell line is associated with degradation of CREB-binding protein. Hum Mol Genet 12:1-12.

Johnson TE, Mitchell DH, Kline S, Kemal R, Foy J (1984) Arresting development arrests aging in the nematode Caenorhabditis elegans. Mech Aging Dev 28:23-40.

Kim JK, Gabel HW, Kamath RS, Tewari M, Pasquinelli A, Rual JF, Kennedy S, 
Dybbs M, Bertin N, Kaplan JM, Vidal M, Ruvkun G (2005) Functional genomic analysis of RNA interference in $C$. elegans. Science 308:1164-1167.

Knoepfler PS, Eisenman RN (1999) Sin meets NuRD and other tails of repression. Cell 99:447-450.

Li J, Wang J, Wang J, Nawaz Z, Liu JM, Qin J, Wong J (2000) Both corepressor proteins SMRT and N-CoR exist in large protein complexes containing HDAC3. EMBO J 19:4342-4350.

Mangiarini L, Sathasivam K, Seller M, Cozens B, Harper A, Hetherington C, Lawton M, Trottier Y, Lehrach H, Davies SW, Bates GP (1996) Exon 1 of the HD gene with an expanded CAG repeat is sufficient to cause a progressive neurological phenotype in transgenic mice. Cell 87:493-506.

Mantamadiotis T, Lemberger T, Bleckmann SC, Kern H, Kretz O, Martin Villalba A, Tronche F, Kellendonk C, Gau D, Kapfhammer J, Otto C, Schmid W, Schutz G (2002) Disruption of CREB function in brain leads to neurodegeneration. Nat Genet 31:47-54.

Mao Z, Wiedmann M (1999) Calcineurin enhances MEF2 DNA binding activity in calcium-dependent survival of cerebellar granule neurons. J Biol Chem 274:31102-31107.

Matsuyama A, Shimazu T, Sumida Y, Saito A, Yoshimatsu Y, SeigneurinBerny D, Osada H, Komatsu Y, Nishino N, Khochbin S, Horinouchi S, Yoshida M (2002) In vivo destabilization of dynamic microtubules by HDAC6-mediated deacetylation. EMBO J 21:6820-6831.

McCampbell A, Taylor JP, Taye AA, Robitschek J, Li M, Walcott J, Merry D, Chai Y, Paulson H, Sobue G, Fischbeck KH (2000) CREB-binding protein sequestration by expanded polyglutamine. Hum Mol Genet 9:2197-2202.

McCampbell A, Taye AA, Whitty L, Penney E, Steffan JS, Fischbeck KH (2001) Histone deacetylase inhibitors reduce polyglutamine toxicity. Proc Natl Acad Sci USA 98:15179-15184.

North BJ, Marshall BL, Borra MT, Denu JM, Verdin E (2003) The human Sir2 ortholog, SIRT2, is an NAD+-dependent tubulin deacetylase. Mol Cell 11:437-444.

Nucifora Jr FC, Sasaki M, Peters MF, Huang H, Cooper JK, Yamada M, Takahashi H, Tsuji S, Troncoso J, Dawson VL, Dawson TM, Ross CA (2001) Interference by huntingtin and atrophin-1 with cbp-mediated transcription leading to cellular toxicity. Science 291:2423-2428.

Parker JA, Arango M, Abderrahmane S, Lambert E, Tourette C, Catoire H, Neri C (2005) Resveratrol rescues mutant polyglutamine cytotoxicity in nematode and mammalian neurons. Nat Genet 37:349-350.

Perkins LA, Hedgecock EM, Thomson JN, Culotti JG (1986) Mutant sensory cilia in the nematode Caenorhabditis elegans. Dev Biol 117:456-487.

Ross CA (2002) Polyglutamine pathogenesis: emergence of unifying mechanisms for Huntington's disease and related disorders. Neuron 35:819-822.

Sakahira H, Breuer P, Hayer-Hartl MK, Hartl FU (2002) Molecular chaperones as modulators of polyglutamine protein aggregation and toxicity. Proc Natl Acad Sci USA 99 [Suppl 4]:16412-16418.

Schweizer E, Hofmann J (2004) Microbial type I fatty acid synthases (FAS): major players in a network of cellular FAS systems. Microbiol Mol Biol Rev 68:501-517.

Shi Y, Mello C (1998) A CBP/p300 homolog specifies multiple differentiation pathways in Caenorhabditis elegans. Genes Dev 12:943-955.

Simmer F, Tijsterman M, Parrish S, Koushika SP, Nonet ML, Fire A, Ahringer J, Plasterk RH (2002) Loss of the putative RNA-directed RNA polymer- ase RRF-3 makes C. elegans hypersensitive to RNAi. Curr Biol 12:1317-1319.

Steffan JS, Bodai L, Pallos J, Poelman M, McCampbell A, Apostol BL, Kazantsev A, Schmidt E, Zhu YZ, Greenwald M, Kurokawa R, Housman DE, Jackson GR, Marsh JL, Thompson LM (2001) Histone deacetylase inhibitors arrest polyglutamine-dependent neurodegeneration in Drosophila. Nature 413:739-743.

Sugars KL, Rubinsztein DC (2003) Transcriptional abnormalities in Huntington disease. Trends Genet 19:233-238.

Tanner KG, Landry J, Sternglanz R, Denu JM (2000) Silent information regulator 2 family of NAD- dependent histone/protein deacetylases generates a unique product, 1-O-acetyl-ADP-ribose. Proc Natl Acad Sci USA 97:14178-14182.

Taylor JP, Taye AA, Campbell C, Kazemi-Esfarjani P, Fischbeck KH, Min KT (2003) Aberrant histone acetylation, altered transcription, and retinal degeneration in a Drosophila model of polyglutamine disease are rescued by CREB-binding protein. Genes Dev 17:1463-1468.

Thatcher JD, Haun C, Okkema PG (1999) The DAF-3 Smad binds DNA and represses gene expression in the Caenorhabditis elegans pharynx. Development 126:97-107.

Timmons L, Court DL, Fire A (2001) Ingestion of bacterially expressed dsRNAs can produce specific and potent genetic interference in Caenorhabditis elegans. Gene 263:103-112.

Tissenbaum HA, Guarente L (2001) Increased dosage of a sir-2 gene extends lifespan in Caenorhabditis elegans. Nature 410:227-230.

Troemel ER, Chou JH, Dwyer ND, Colbert HA, Bargmann CI (1995) Divergent seven transmembrane receptors are candidate chemosensory receptors in C. elegans. Cell 83:207-218.

Victor M, Bei Y, Gay F, Calvo D, Mello C, Shi Y (2002) HAT activity is essential for CBP-1-dependent transcription and differentiation in Caenorhabditis elegans. EMBO Rep 3:50-55.

Wen YD, Perissi V, Staszewski LM, Yang WM, Krones A, Glass CK, Rosenfeld MG, Seto E (2000) The histone deacetylase-3 complex contains nuclear receptor corepressors. Proc Natl Acad Sci USA 97:7202-7207.

Whetstine JR, Ceron J, Ladd B, Dufourcq P, Reinke V, Shi Y (2005) Regulation of tissue-specific and extracellular matrix-related genes by a class I histone deacetylase. Mol Cell 18:483-490.

Wood JG, Rogina B, Lavu S, Howitz K, Helfand SL, Tatar M, Sinclair D (2004) Sirtuin activators mimic caloric restriction and delay ageing in metazoans. Nature 430:686-689.

Youn HD, Sun L, Prywes R, Liu JO (1999) Apoptosis of T cells mediated by $\mathrm{Ca}^{2+}$-induced release of the transcription factor MEF2. Science 286:790-793.

Youn HD, Grozinger CM, Liu JO (2000) Calcium regulates transcriptional repression of myocyte enhancer factor 2 by histone deacetylase 4 . J Biol Chem 275:22563-22567.

Zhang S, Sokolchik I, Blanco G, Sze JY (2004) Caenorhabditis elegans TRPV ion channel regulates $5 \mathrm{HT}$ biosynthesis in chemosensory neurons. Development 131:1629-1638.

Zhang Y, Li N, Caron C, Matthias G, Hess D, Khochbin S, Matthias P (2003) HDAC-6 interacts with and deacetylates tubulin and microtubules in vivo. EMBO J 22:1168-1179.

Zoghbi HY, Orr HT (2000) Glutamine repeats and neurodegeneration. Annu Rev Neurosci 23:217-247. 